\title{
Effects of Short-term Huatou Chan Training on Health
}

Abstract Previous studies have shown that perennial Chan training leads to improvements in brain functioning. However, few studies have investigated the effects of short-term Huatou Chan training. The current study explored the effects of a three-day Huatou Chan training on physical and emotional health, as well as brain state. Seventy healthy subjects were recruited and divided into two groups: the Huatou Chan group and the Control group. The Huatou Chan group received a 3-day Huatou Chan training, while the Control group waited for three days. Both groups completed a 6-min Brain State Index recording, the SCL-90, the Brief Profile of Mood State, the Meaning in Life Questionnaire, and the Index of Well-being, prior to and after the training or waiting period. Results showed that short-term Huatou Chan training had significant benefits on physical and emotional health (somatization, obsessive-compulsive, depression, hostility, and psychoticism), negative emotions (tension-anxiety, depression-dejection, anger-hostility, fatigue-inertia, and confusion-bewilderment), well-being, and attitude towards life. In addition, short-term Chan training can significantly improve brain state, as shown by the index of depression, anxiety, alerting, intelligence, and sleep disorder. To our knowledge, this is the first study to provide direct evidence for the benefits of short-term intensive Huatou Chan training on physical and mental health.

Key words: Short-term; Huatou Chan; meditation; health; brain activity

\section{Introduction}

In recent decades, the study of meditation has attracted the attention of many scholars (Lutz, Slagter, Dunne, \& Davidson, 2008; Sedlmeier et al., 2012; Tomlinson, Yousaf, Vitterso, \& Jones, 2018). Meditation refers to a family of self-regulation practices that focus on training attention and awareness to bring mental processes under greater voluntary control. Meditation can thereby foster general mental well-being and the development of specific capacities such as calmness, clarity, and concentration (Walsh \& Shapiro, 2006). Generally, meditation is grouped into two broad categories: focused attention (FA) and open monitoring (OM). The first category, FA meditation, entails voluntary concentration on an object in a sustained manner. OM meditation, on the other hand, involves non-reactive monitoring of the content of experience from moment to moment (Lutz et al., 2008).

At present, a large number of studies have shown that meditation training can significantly improve individual health (Lynch, Gander, Nahar, Kohls, \& Walach, 2018; Sedighimornani, Rimes, \& Verplanken, 2019; Tomlinson et al., 2018; Y. Wang \& Luo, 2017) and cognitive ability (Chiesa, Calati, \& Serretti, 2011; Lutz et al., 2008). For example, studies showed that the quality of mindfulness (which can be improved by meditation) regulates mood by improving emotional resilience (Y. Wang, Xu, \& Luo, 2016). Mindfulness-based coping program could help university students bring mindful awareness to their academic work, stress management, approach to communication and relationships, and health (Lynch et al., 2018). In other studies, it has been revealed that mindfulness was associated with compassion (Sedighimornani et al., 2019), and meditation training significantly increases individual pain tolerance (Y. Wang et al., 2019).

Despite the rapid development of meditation research, there are relatively few studies on the effects of Chan meditation. In Eastern culture, Chan is a common type of meditation. Indeed, the 
Chinese term Chan (Zen in Japanese) is an abbreviation of the Sanskrit term dhyäna, which is similar to the modern English word "meditation". Chan originated in Bodhidharma (around 440-520), flourished in the Sixth Patriarch Huineng (around 638-713), and became the mainstream of Buddhism in the middle and late Tang Dynasty (618-907). After the Song Dynasty (960-1279), Chinese Chan spread throughout East Asia and Southeast Asia, giving birth to the various Zen, Sõn, and Thien lineages of Japan, Korea, and Vietnam, respectively (Sharf, 2014). Today these traditions continue to promote the study of meditation and have attracted the attention of many scholars.

There are differences between Chan and other forms or Western cultural meditation. First, from a training perspective, the main characteristic of Chan is koan or huatou practice which focuses on cryptic utterances of past masters (Sharf, 2014). Koan may be a story, a question, or a word. It requires refraining from thoughts, memories, emotions, associations, and perceptions while maintaining consciousness (Hinterberger, Schmidt, Kamei, \& Walach, 2014). In contrast, meditation training by Western scholars is mostly derived from mindfulness, in which practitioners are instructed to focus on internal and external feelings with acceptance and awareness (Kabat-Zinn, 1990). Second, while the goal of Chan practitioners is to eventually achieve enlightenment (Sharf, 2014), the goal of mindfulness practices is to calm the mind and to reduce distractions. As focused attention advances, well-developed monitoring skills become the main point of transition into mindfulness practice (Lutz et al., 2008). Third, electrophysiological brain activity during Chan is different from that during other meditation training. For example, Hinterberger et al. (2014) compared electrophysiological activity during "thoughtless emptiness" (TE, a state evoked by Huatou Chan), FA and OM. The results of the study showed a significant decrease in central and parietal gamma waves during a TE task compared to an FA task. In addition, there were decreased alpha and beta amplitudes, mainly in parietal areas, during TE compared to OM. Meanwhile, TE also presented significantly less delta and theta waves than a wakeful, closed eyes, resting condition (Hinterberger et al., 2014).

At present, there are limited exploratory studies on Chan. One study found that experienced meditators (with an average practice time of $18.4 \pm 2.6$ years) exhibit a continuously high degree of cardiorespiratory phase synchronization (Chang \& Lo, 2013). Lo and Chang (2013) found that Chan experts (average practice time of 5.8 years) display enhanced interaction of local neural networks during Chan state compared to resting state. In another study, Lin, Chang, Zemon, and Midlarsky (2007) investigated the effects of 8-weeks of Chan meditation practice on musical performance anxiety and musical performance quality but found no effect of the meditation training on quality of musical performance.

There remain several questions to be answered in the study of Chan meditation. First, existing research has mainly focused on cross-sectional studies comparing expert meditators to control groups (Chang \& Lo, 2013; Lo \& Chang, 2013). Due to the study design, it is difficult to exclude various confounding factors such as baseline differences and motivation. The only short-term Chan training study to date found no significant improvement in behavioral performance (Lin et al., 2007). Second, the content of Chan training varies from study to study. For example, some studies emphasize the conversion of chest breathing to umbilical chakra breathing and into deep meditation (Chang \& Lo, 2013; Lo \& Chang, 2013), while other studies focus on the training of body and room awareness, as well as behavioral visualization training and psychological rehearsal (Lin et al., 2007). To date, there are no reported studies on Huatou Chan. 
Third, previous studies have used small sample sizes, limiting the generalization of conclusions (Chang \& Lo, 2013; Lin et al., 2007; Lo \& Chang, 2013). Last, the existing research is limited to studies of specific abilities (such as the quality of musical performance), and therefore lacks a comprehensive exploration of the effects of Chan on physical and mental health (Lin et al., 2007).

In the current study, we recruited a large sample of participants and conducted a 3-day short-term Huatou Chan meditation training using a controlled experimental design. The effects of Huatou Chan meditation training on physical and mental health were explored from the aspects of relieving physical symptoms, regulating emotions, improving well-being, changing life attitudes and monitoring brain status.

\section{Method}

\section{Participants}

A power analysis using G*Power 3.1 (the University of Trier, Germany) revealed that 54 participants are required to detect a time $\times$ group interaction with $95 \%$ power (ANOVA: repeated measures, within - between interaction; at the 0.05 significance level; Faul, Erdfelder, Lang, \& Buchner, 2007). The analysis was based on the assumption of a small-medium effect size ( $\mathrm{f}=$ 0.25 ), established by a recent meta-analysis of mindfulness intervention (Eberth \& Sedlmeier, 2012). Taking dropout rate into consideration, we oversampled and recruited 70 participants. The subjects were recruited through introductory lectures open to the general public. The theme of the lecture was "Chan: The way to reduce stress and improve well-being". Thirty subjects participated in the Huatou Chan training group, and another forty subjects were recruited as controls. Finally, 61 subjects completed the pre-test and post-test (Huatou Chan group: 21 subjects, Mean age = $44.47 \pm 8.02$ years; Control group: 40 subjects, Mean age $=46.33 \pm 8.28$ years). All participants signed a written informed consent prior to participation. The experimental protocol was approved by the institutional review board of Institute of Psychology, Chinese Academy of Sciences.

\section{Materials and measures}

Symptom Checklist 90 (SCL-90) The Symptom Checklist-90 (SCL-90) is a self-reported questionnaire on symptoms and psychopathologic features. There are 10 subscales derived from the SCL-90: somatization, obsessive-compulsive, interpersonal sensitivity, anxiety, depression, hostility, phobic anxiety, paranoid ideation, psychoticism, and additional symptoms. The items are rated on a scale from 1 to 5 . The SCL-90 has been used extensively in studies of various mental disorders, with medical and psychiatric inpatients and outpatients, in clinical drug trials, and in community surveys (Derogatis \& Cleary, 1977). In the current study, the Cronbach's alphas of the subscales were $0.662-0.874$.

Brief Profile of Mood State (BPOMS) The Brief Profile of Mood State is a 30-item inventory (Chi \& Lin, 2003) consisting of five subscales: tension-anxiety (e.g., Tense); depression-dejection (e.g., Sorry for things done); anger-hostility (e.g., Furious); vigor-activity (e.g., Active); fatigue-inertia (e.g., Fatigued); and confusion-bewilderment (e.g., Forgetful). A total mood disturbance score (TMD) was calculated by adding the five subscales. In the current study, the Cronbach's alphas of the subscales were $0.727-0.915$.

Meaning in Life Questionnaire (MLQ) The Meaning in Life Questionnaire is a 10-item questionnaire (Steger, Frazier, Oishi, \& Kaler, 2006; M. Wang \& Dai, 2008). Items are rated on a 7-point scale ranging from 1 (absolutely untrue) to 7 (absolutely true). The MLQ consists of two 
subscales: The presence of meaning in life (e.g. I understand my life's meaning), and the search for meaning of life (I am looking for something that makes my life feel meaningful). In the current study, the Cronbach's alphas of the subscales were 0.793 and 0.844 .

Index of Well-being (IWB) The Index of Well-being is a 9-item scale (Campbell, Converse, \& Rodgers, 1976). Each item is rated on a 7-point scale. In the current study, the Cronbach's alpha of the scale was 0.759 .

Index of Brain State Recording (Luo et al., 2009; Ren et al., 2011) The index of brain state was recorded by the portable HXD-I multi-functional combined monitor (Yi Fei Hua Tong Technology Development co., LTD., Beijing). Electrode impedance was reduced to less than $5 \mathrm{k} \Omega$. The signal was referenced on A1, A2 and FPZ. The electrodes used were FP1 and FP2.

The resolution of EEG signal acquisition was $1 \mathrm{~ms}$ and the acquisition and processing window was 1.25 seconds. The data acquisition accuracy was 12 bits. Each measurement was six minutes long. Indices of brain state recording included the index of depression, anxiety, alerting, intelligence, and sleep disorder. Previous studies have confirmed that brain state indicators can be used to monitor a normal subject's state of concentration, wakefulness and relaxation (Ren et al., 2011). Brain state measurements can also be used as real-time biofeedback indicators for clinical samples, such as was the case for psychological rehabilitation after the Wenchuan earthquake (Luo et al., 2009).

\section{Training protocol}

The training was initiated and guided by a Huatou Chan master (the $2^{\text {nd }}$ author) and his assistants (the $5^{\text {th }}$ and $6^{\text {th }}$ authors), who are sponsors of the Universal Scientific Chanism Meditation System (USCMS). A 3-day Scientific Chan Meditation training was held in Tai' an, Shandong Province, China. There were six 45-minute sessions each day. Prior to training, the Huatou Chan master conducted one-on-one interviews with participants to establish the focus of training. The Huatous were derived from three main categories, abbreviated as "what", "why" and "how". These condensed words can stem from various questions such as What on earth is the meaning of life? Why am I always so angry? And How should I solve the problem? During the 3-day training, participants were asked to focus on her/his Huatou.

During each session, participants closed their eyes and were instructed to adjust the body, breath, and mind. Participants were then asked to scrutinize their "Huatou". That is, under the guidance of the Huatou Chan master, participants meditated on their own Huatou, searched for answers, denied the answers, and searched again. Huatou is a process of self-denial. During this period, the Huatou Chan master adjusted the participant's state accordingly. When the Huatou Chan master found that a participant was holding on to the Huatou tightly, and reaching the critical state, the Huatou Chan master immediately performed the blow and shout, that is, the Huatou Chan master and his assistants would shout loudly at the participant about the Huatou, accompanied by a sharp bah! The purpose of the blow and shout technique is to help participants to further engage in the Huatou. When a participant is fully focused on the Huatou and the application of the blow and shout technique is resonant, a breakthrough state is achieved.

\section{Study procedures}

Participants in both the Huatou and Control groups completed the Index of Brain State Recording, and the SCL-90, the BPOMS, the MLQ, and the Index of Well-being. The Huatou Chan group then participated in a 3-day Huatou Chan training, while the Control group waited for 
three days.

\section{Data analysis}

Statistical analyses were performed with SPSS 16.0 (SPSS Software, Armonk, NY, USA), and GraphPad Prism 5.0 (GraphPad Software, La Jolla, CA, USA). Statistical significance was set at $p<0.05$.

Group differences in gender were analyzed by Chi-square analysis. Group differences in age were analyzed by an independent $t$-test. The differences in questionnaire scores between pre-test and post-test were compared between groups using independent $t$-tests.

The collected EEG signals from the prefrontal lobe were amplified, filtered and converted from $\mathrm{A} / \mathrm{D}$ to digital signals to obtain the time domain EEG data. Next, the metadata of regular feature changes in EEG were obtained by applying the methods of wavelet analysis, spectral analysis and pattern recognition analysis. Finally, the metadata were used as predictive variables and brain status was the dependent variable in a multivariate regression analysis to find the relationship between metadata and brain state. The final index of brain state was obtained after weighted normalization ( $\mathrm{Wu}, 2017)$.

\section{Results}

\section{Preliminary analyses}

A Chi-square test and an independent $t$-test, respectively, showed no significant differences between groups in gender distribution $\left(\chi^{2}=0.115, p=0.735\right)$ or age $(t=-0.810, p=0.421)$. Differences in pre- to post-test changes in questionnaire scores between groups are shown in Table 1.

\section{Alterations in physical and mental symptoms}

Independent sample $t$ - tests revealed significant differences between the Huatou Chan group and the Control group on SCL-90 symptoms, indicating that those receiving Huatou Chan training showed a significantly greater decrease in symptoms from pre-test to post-test ( $M$ change $=$ 12.43), than those in the Control group ( $M$ change $=-2.23$ ), $t=-2.941, p=0.005$. Significant differences were also found on SCL-90 total score, with the Huatou Chan group showing a greater decrease $(M$ change $=-25.52)$ than the control group $(M$ change $=-6.45), t=-2.301, p=0.025$ between pre-test and post-test. In addition, those receiving Huatou Chan training showed a significantly greater decrease from pre-test to post-test than those in the Control group in symptom subscales of somatization ( $M$ change: -3.33 vs. $-0.13, t=-2.119, p=0.038$ ), obsessive-compulsive ( $M$ change: -3.33 vs. $-0.20, t=-2.515, p=0.015)$, depression ( $M$ change: -4.57 vs. $-1.43, t=$ $-2.331, p=0.023$ ), hostility ( $M$ change: -2.14 vs. $-0.30, t=-2.623, p=0.011$ ), and psychoticism ( $M$ change: -3.05 vs. $-0.63, t=-2.504, p=0.015$ ). These results indicate that physical and psychological symptoms among individuals are alleviated to a greater extent after Chan training than in the Control condition with no training. No other SCL-90 symptom scores showed significant differences between groups. See Table 1 for a summary of independent sample $t$-test results.

There were significant differences between the Huatou Chan group and the control group on the BPOMS scale. As expected, there was a greater reduction in negative emotion ratings in the Huatou Chan group compared to the Control group. The Huatou Chan group showed greater decreases between pre- and post-test than the Control group in total mood disturbance ( $M$ change: -13.57 vs. $-0.83, t=-3.378, p=0.001)$, tension-anxiety ( $M$ change: -2.62 vs. $-0.20, t=-3.560, p=$ 
0.001 ), depression-dejection ( $M$ change: -3.19 vs. $-0.48, t=-2.494, p=0.015)$, anger-hostility $(M$ change: -3.19 vs. $-0.63, t=-3.151, p=0.003$ ), fatigue-inertia ( $M$ change: -2.71 vs. $-0.13, t=$ $-2.530, p=0.014)$, and confusion-bewilderment ( $M$ change: -1.86 vs. $-0.20, t=-2.604, p=0.012$ ). These results indicate that participants in the Huatou Chan group were able to effectively decrease negative emotions. However, there were no significant changes in vigor-activity. Table 1 summarizes the independent sample $t$-test results.

\section{Changes in meaning of life and well-being}

The score on the search for meaning in life subscale of the MLQ, was reduced from pre- to post-test to a greater extent in the Chan group compared to the Control group ( $M$ change: -5.24 vs. $0.08, t=-3.333, p=0.001)$. However, between-group analysis found no significant difference between the Chan group and Control groups in presence of meaning in life subscale $(t=1.323, p$ $=0.191$ ). These results indicate that individuals that received Chan training were more aware and less confused about of the meaning of life after training.

There was a significant difference between groups in the change in IWB score from pre-test to post-test. The Chan training group showed an increase in IWB score ( $M$ change: 4.47$)$, while the Control group showed a decrease is IWB score ( $M$ change: -0.87 ), $t=2.264, p=0.027$. This result indicates improved well-being in the Chan training condition, while those in the control condition demonstrated diminished well-being over time.

\section{Alterations in brain state}

There were significant group differences in brain state changes between the pre-test and the post-test. The Huatou Chan training group demonstrated decreased brain activity associated with depression $(t=5.554, p<0.001)$, anxiety $(t=5.139, p<0.001)$, alertness $(t=2.571, p=0.006)$, and sleep disorder $(t=2.101, p=0.047)$, while showing greater brain activity associated with the index of intelligence $(t=-4.822, p<0.001)$. These results indicate that Huatou Chan training tends to reduce anxiety, depression, and alertness, while developing intelligence and improving sleep quality.

\section{Discussion}

Previous studies have shown that perennial Chan training improves brain activity. However, it is difficult to exclude confounding factors such as baseline differences and motivation between Chan experts and controls in cross-sectional studies. Therefore, the current study explored the effects of short-term Chan meditation training. In addition, the current study is the first to examine Huatou Chan training specifically. Moreover, previous studies used small sample sizes, and focused on one particular ability, limiting the generalization of conclusions. The current study is the first to compare the effects of a three-day Huatou Chan meditation on physical and mental health, and brain state. The results show that short-term Huatou Chan meditation training can significantly reduce physical and mental symptoms, reduce negative emotions, enhance well-being and reduce the pursuit of meaning in life. In addition, short-term Huatou Chan meditation training can significantly improve brain states in indices of depression, anxiety, alertness, intelligence, and sleep disorder.

We can deepen our understanding of meditation by distinguishing Huatou Chan from other meditation types and verifying the effects of Huatou Chan. The current study demonstrated that 
systematic short-term Huatou Chan training can significantly improve physical and mental health, similar to the effects of mindfulness training (Chiesa et al., 2011; Y. Wang et al., 2019; Y. Wang et al., 2016). Prior studies have found that mindfulness training can regulate negative emotions by reducing mind wandering (Y. Wang, Xu, Zhuang, \& Liu, 2017). Through the process of Huatou Chan meditation, practitioners are taught to refrain from thoughts, memories, emotions, associations, and perceptions while maintaining consciousness. As a result, mental processing is reduced (Hinterberger et al., 2014). Therefore, Chan practitioners may be less troubled by negative emotions or thoughts (e.g. obsessive-compulsive thoughts, hostility thoughts).

A prior meta-analysis found limited evidence for improved mental health-related quality of life from meditation programs (Goyal et al., 2014). However, it should be noted that this is the first study to examine Huatou Chan meditation. Training content and objectives, along with brain activity, differ between mindfulness training and Huatou Chan training (Kabat-Zinn, 1990; Lutz et al., 2008; Sharf, 2014). Huatou Chan also differs from previous Chan training studies that emphasize the conversion of chest breathing into umbilical chakra breathing and into deep meditation, or that focus on the training of body and room awareness, behavioral visualization training, or psychological rehearsal (Chang \& Lo, 2013; Lin et al., 2007; Lo \& Chang, 2013). The current study found alterations in one's attitude towards life with Huatou Chan training. These results demonstrate that the Huatou Chan practitioner reduces the impulse to pursue the meaning of life (for example, "I am looking for something that makes my life feel meaningful"). Importantly, their experience of life has not been affected (for example, "I know the meaning of my life"), indicating that Huatou Chan reduces the individual's confusion about life. This may be one of the important differences between Huatou Chan and other meditation types. The content of Huatou is closely related to problems and dilemmas in life. The process of Huatou Chan training is the silent recitation of a Huatou until the final "satori" is attained. With a blow and shout at the right time, practitioners no longer focus on the complexities of life, and ultimately achieve enlightenment.

In a previous study, Lin et al. (2007) tested the effect of an 8-week Chan training on musical performance anxiety and musical performance quality. In the study, participants practiced body and room awareness, as well as behavior visualization and psychological rehearsal. The results showed that 8 weeks of meditation training did not significantly improve the level of music performance. In addition, nearly $40 \%$ of the participants in the Chan meditation group dropped out of the study for various reasons. The effectiveness of short-term Huatou Chan meditation training found in the present study (as opposed to the study described above) may be due to the following. First, the Huatou Chan Master played an important role in training. Prior to training, the Huatou Chan Master conducted detailed one-on-one interviews with practitioners, establishing the content of the Huatou. Second, during training, the Huatou Chan Master performed a blow and a shout at appropriate times. Third, intensive short-term training may be more suitable for Chan training, because practitioners can continuously concentrate on the Huatou.

The current study has some limitations that should be considered. First, the experimental results may be affected by demand characteristics. In future studies, randomized controlled trials including a positive control group should be used to compare other training methods with Huatou Chan. Second, the participants in the current study were mostly middle-aged, and as such caution must be used when making generalizations to other age groups. Despite these limitations, the current study is the first to provide evidence for short-term Huatou Chan meditation training on 
improved health. The results show that short-term Huatou Chan meditation training has significant benefits on physical and mental health, as well as brain activity.

\section{Ethics Statement}

The study received ethical approval from the Institute of Psychology, Chinese Academy of Sciences. No adverse events were reported in this study.

Informed Consent

Informed consent was obtained from all individual participants. 
Table 1. Differences in pre- to post-test changes in questionnaire scores between groups $(M \pm S D)$

\begin{tabular}{lcccc}
\hline Variables & Chan group $(\mathrm{n}=21)$ & Control group $(\mathrm{n}=40)$ & $t$ & $p$ \\
\hline SCL-90 & & & & \\
Number of Positive symptoms & $-12.43 \pm 17.47$ & $-2.23 \pm 9.71$ & -2.941 & $\mathbf{0 . 0 0 5}$ \\
SCL-90 total score & $-25.52 \pm 45.99$ & $-6.54 \pm 17.46$ & -2.301 & $\mathbf{0 . 0 2 5}$ \\
Somatization & $-3.33 \pm 9.01$ & $0.13 \pm 3.58$ & -2.119 & $\mathbf{0 . 0 3 8}$ \\
Obsessive-compulsive & $-3.33 \pm 5.91$ & $-0.20 \pm 3.80$ & -2.515 & $\mathbf{0 . 0 1 5}$ \\
Interpersonal sensitivity & $-2.95 \pm 4.78$ & $-1.48 \pm 2.54$ & -1.582 & 0.119 \\
Depression & $-4.57 \pm 7.43$ & $-1.43 \pm 3.11$ & -2.331 & $\mathbf{0 . 0 2 3}$ \\
Anxiety & $-2.71 \pm 7.56$ & $-0.78 \pm 3.40$ & -1.384 & 0.172 \\
Hostility & $-2.14 \pm 3.47$ & $-0.30 \pm 2.03$ & -2.623 & $\mathbf{0 . 0 1 1}$ \\
Photic anxiety & $-0.38 \pm 2.96$ & $-0.30 \pm 2.58$ & -0.111 & 0.912 \\
Paranoid ideation & $-1.57 \pm 3.56$ & $-0.80 \pm 1.92$ & -1.103 & 0.275 \\
Psychoticism & $-3.05 \pm 4.88$ & $-0.63 \pm 2.70$ & -2.504 & $\mathbf{0 . 0 1 5}$ \\
Additional items & $-1.48 \pm 3.40$ & $-0.85 \pm 2.36$ & -0.843 & 0.403 \\
BPOMS & & & & \\
Total mood disturbance & $-13.57 \pm 20.46$ & $-0.83 \pm 9.04$ & -3.378 & $\mathbf{0 . 0 0 1}$ \\
Tension-anxiety & $-2.62 \pm 3.84$ & $-0.20 \pm 2.34$ & -3.560 & $\mathbf{0 . 0 0 1}$ \\
Depression-dejection & $-3.19 \pm 5.60$ & $-0.48 \pm 2.94$ & -2.494 & $\mathbf{0 . 0 1 5}$ \\
Anger-hostility & $-3.19 \pm 4.25$ & $-0.63 \pm 2.13$ & -3.151 & $\mathbf{0 . 0 0 3}$ \\
Vigor-activity & $-0.76 \pm 4.72$ & $0.20 \pm 3.33$ & -0.925 & 0.359 \\
Fatigue-inertia & $-2.71 \pm 5.34$ & $-0.13 \pm 2.68$ & -2.530 & $\mathbf{0 . 0 1 4}$ \\
Confusion-bewilderment & $-1.86 \pm 3.57$ & $0.20 \pm 2.54$ & -2.604 & $\mathbf{0 . 0 1 2}$ \\
MLQ & & & & \\
Presence of meaning in life & $2.43 \pm 7.37$ & $0.13 \pm 5.94$ & 1.323 & 0.191 \\
Search for meaning of life & $-5.24 \pm 5.97$ & $0.08 \pm 5.89$ & -3.333 & $\mathbf{0 . 0 0 1}$ \\
IWB & & & & \\
Index of well-being & $4.47 \pm 9.33$ & $-0.87 \pm 8.43$ & 2.264 & $\mathbf{0 . 0 2 7}$ \\
\hline & & & &
\end{tabular}



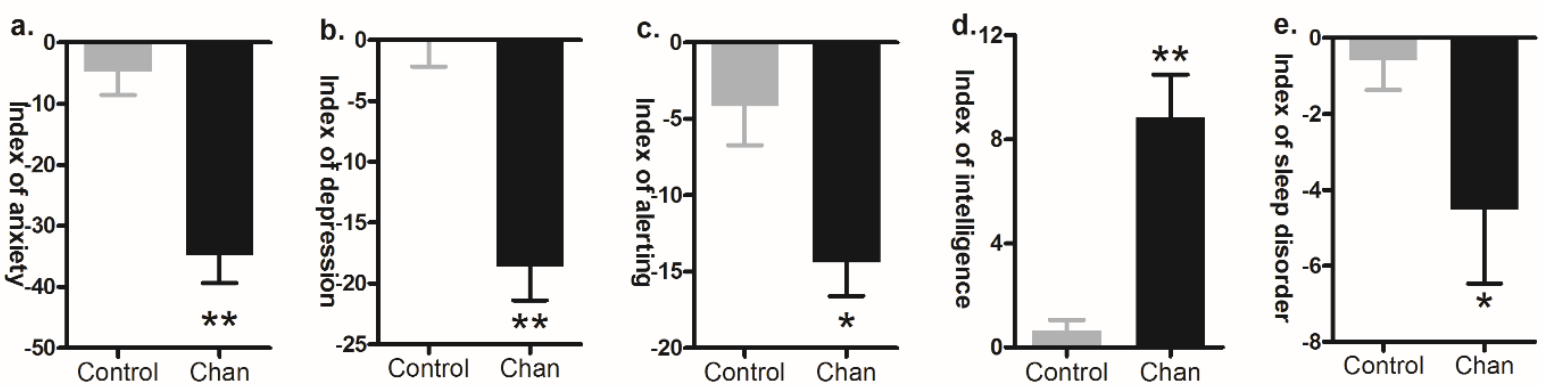

Figure 1. Improvement in brain state after Chan training. ${ }^{*} p<0.05 ;{ }^{* *} p<0.01$. 


\section{Reference}

Campbell, A., Converse, P. E., \& Rodgers, W. L. (1976). The quality of American life: Perceptions, evaluations, and satisfactions. American Journal of Sociology, 6(6), 694.

Chang, C. H., \& Lo, P. C. (2013). Effects of long-term dharma-chan meditation on cardiorespiratory synchronization and heart rate variability behavior. Rejuvenation Research, 16(2), 115-123. doi: $10.1089 /$ rej.2012.1363

Chi, S., \& Lin, W. (2003). The Preliminary revision of Brief Profile of Mood States (BPOMS)-Chinese Edition. Chinese Mental Health Journal, 17(11), 768-770.

Chiesa, A., Calati, R., \& Serretti, A. (2011). Does mindfulness training improve cognitive abilities? A systematic review of neuropsychological findings. Clinical Psychology Review, 31(3), 449-464. doi: 10.1016/j.cpr.2010.11.003

Derogatis, L. R., \& Cleary, P. A. (1977). Confirmation of the dimensional structure of the SCL-90: A study in construct validation. Journal of Clinical Psychology, 33(4), 981-989.

Eberth, J., \& Sedlmeier, P. (2012). The Effects of Mindfulness Meditation: A Meta-Analysis. Mindfulness, 3(3), 174-189. doi: 10.1007/s12671-012-0101-x

Faul, F., Erdfelder, E., Lang, A. G., \& Buchner, A. (2007). G*Power 3: A flexible statistical power analysis program for the social, behavioral, and biomedical sciences. Behavior Research Methods, 39(2), 175-191.doi: 10.3758/bf03193146

Goyal, M., Singh, S., Sibinga, E. M., Gould, N. F., Rowland-Seymour, A., Sharma, R., . . . Haythornthwaite, J. A. (2014). Meditation programs for psychological stress and well-being: a systematic review and meta-analysis. JAMA Internal Medicine, 174(3), 357-368. doi: 10.1001/jamainternmed.2013.13018

Hinterberger, T., Schmidt, S., Kamei, T., \& Walach, H. (2014). Decreased electrophysiological activity represents the conscious state of emptiness in meditation. Frontiers in Psychology, 5, 99. doi: 10.3389/fpsyg.2014.00099

Kabat-Zinn, J. (1990). Full Catastrophe Living: How to cope with stress, pain and illness using mindfulness meditation.

Lin, P., Chang, J., Zemon, V., \& Midlarsky, E. (2007). Silent illumination: a study on Chan (Zen) meditation, anxiety, and musical performance quality. Psychology of Music, 36(2), 139-155. doi: $10.1177 / 0305735607080840$

Lo, P. C., \& Chang, C. H. (2013). Spatially Nonlinear Interdependence of Alpha-Oscillatory Neural Networks under Chan Meditation. Evidence - Based Complementary and Alternative Medicine, 2013,360-371.doi: 10.1155/2013/360371

Luo, F., Luo, J., Wu, Y., Li, J., Wang, J., \& Guo, J. (2009). Psychosomatic recovery by relaxing and rebuilding the brain: Possible application in post traumatic psychological recovery. Advances in Psychological Science, 17(3), 594-601.

Lutz, A., Slagter, H. A., Dunne, J. D., \& Davidson, R. J. (2008). Attention regulation and monitoring in meditation. Trends in Cognitive Sciences, 12(4), 163-169. doi: 10.1016/j.tics.2008.01.005

Lynch, S., Gander, M.-L., Nahar, A., Kohls, N., \& Walach, H. (2018). Mindfulness-Based coping with university life: A randomized wait-list controlled study. SAGE Open, 8(1), 215824401875837. doi: $10.1177 / 2158244018758379$

Ren, J., Huang, Z., Luo, J., Wei, G., Ying, X., Ding, Z., . . . Luo, F. (2011). Meditation promotes insightful problem-solving by keeping people in a mindful and alert conscious state. Science China Life Sciences, 54(10), 961-965. 
Sedighimornani, N., Rimes, K. A., \& Verplanken, B. (2019). Exploring the relationships between mindfulness, self-compassion, and shame. SAGE Open, doi: 10.1177/2158244019866294

Sedlmeier, P., Eberth, J., Sch warz, M., Zimmermann, D., Haarig, F., Jaeger, S., \& Kunze, A. S. (2012). The psychological effects of meditation: A meta-Analysis. Psychological Bulletin, 138(6), 1139-1171. doi: 10.1037/a0028168.supp

Sharf, R. (2014). Mindfulness and Mindlessness in Early Chan (Vol. 64): University of Hawai'i Press.

Steger, M. F., Frazier, P., Oishi, S., \& Kaler, M. (2006). The meaning in life questionnaire: Assessing the presence of and search for meaning in life. Journal of Counseling Psychology, 53(1), 80-93. doi: 10.1037/0022-0167.53.1.80

Tomlinson, E. R., Yousaf, O., Vitterso, A. D., \& Jones, L. (2018). Dispositional mindfulness and psychological health: a systematic review. Mindfulness, 9(1), 23-43. doi: 10.1007/s12671-017-0762-6

Walsh, R., \& Shapiro, S. L. (2006). The meeting of meditative disciplines and Western psychology: a mutually enriching dialogue. American Psychologist, 61(3), 227-239. doi: 10.1037/0003-066X.61.3.227

Wang, M., \& Dai, X. (2008). Chinese Meaning in Life Questionnaire Revised in college students and its reliability and validity test. Chinese Journal of Clinical Psychology, 16(5), 459-461.

Wang, Y., \& Luo, F. (2017). Effectiveness of short-term meditation: Progress, issues and prospects. Chinese Journal of Clinical Psychology, 25(6), 1184-1190.

Wang, Y., Qi, Z., Hofmann, S. G., M., S., Liu, X., \& Xu, W. (2019). Effect of acceptance versus attention on pain tolerance: dissecting two components of mindfulness. Mindfulness. doi: 10.1007/s12671-019-1091-8

Wang, Y., Xu, W., \& Luo, F. (2016). Emotional resilience mediates the relationship between mindfulness and emotion. Psychological Reports, 118(3), 725-736. doi: $10.1177 / 0033294116649707$

Wang, Y., Xu, W., Zhuang, C., \& Liu, X. (2017). Does mind wandering mediate the association between mindfulness and negative mood? A preliminary study. Psychological Reports, 120(1), 118-129. doi: $10.1177 / 0033294116686036$

Wu, Y. (2017). Extraction of objective and quantitative indexes of pain, anxiety,depression and other brain function states from electroencephalogram. China Medical Engineering, 25(4), 1-7. doi: 10.19338/j.issn.1672-2019.2017.04.001 